\title{
Análisis de las condiciones de emprendimiento en los distritos de San Ignacio y Sabanillas del Cantón de Acosta en Costa Rica
}

\section{Analysis of entrepreneurship conditions in the districts of San Ignacio and Sabanillas of Cantón in Acosta, Costa Rica}

\author{
Silvia Sáenz-León ${ }^{1}$ https://orcid.org/0000-0003-0801-8224, \\ Juan Manuel Monge-Rodríguez ${ }^{2}$ https://orcid.org/0000-0002-0507-1110 \\ ${ }^{1}$ Consejo Nacional de Rectores, San José, Costa Rica \\ ssaenzeconare.ac.cr \\ ${ }^{2}$ Universidad Nacional, Heredia, Costa Rica \\ juanmr09ehotmail.com
}

\section{(c) $(1)(9$}

Esta obra está bajo una licencia internacional

Creative Commons Atribución-NoComercial 4.0.

\section{Resumen}

La presente investigación tiene como objetivo el estudio de las características socioeconómicas y territoriales de algunos emprendimientos provenientes de los distritos de San Ignacio y Sabanillas, cantón Acosta-Costa Rica. Para ello, se realizó una encuesta dirigida a un grupo de emprendedores, utilizando el método muestral denominado "bola de nieve". Esta modalidad permitió identificar diversos aspectos de dichos emprendimientos, como, por ejemplo, los años de funcionamiento y las condiciones de formalidad o semiformalidad en las que estos se han desarrollado. Se obtuvo, asimismo, información sobre esas iniciativas de negocio y sus emprendedores, tales como el género de estos últimos, la cantidad de personas que emplean, la existencia o no de apoyo gubernamental o sobre si estos negocios constituyen la principal fuente de subsistencia familiar para sus gestores y, en caso de que hubiesen contraído algún crédito, identificar su nivel de endeudamiento. Por otra parte, se averiguó acerca del tipo de clientes que acceden a sus ofertas o los efectos que experimentaron a raíz de la irrupción del COVID-19. Los resultados evidenciaron que son las mujeres jóvenes y tituladas, de entre 25 a 33 años, quienes impulsan estos muy variados emprendimientos.

Palabras clave: economía solidaria; emprendimiento femenino; desarrollo local; crecimiento endógeno.

Sumario: Introducción, Materiales y Métodos, Resultados, Discusión de Resultados y Conclusiones.

Como citar: Sáenz-León, S. \& Monge-Rodríguez, J. (2021). Análisis de las condiciones de emprendimiento en los distritos de San Ignacio y Sabanillas del Cantón de Acosta en Costa Rica. Revista Tecnológica -

Espol, 33(3), 68-84. http://www.rte.espol.edu.ec/index.php/tecnologica/article/view/881 


\begin{abstract}
The objective of this research is to study of the socio-economic and territorial characteristics of some enterprises from the districts of San Ignacio and Sabanillas, canton Acosta-Costa Rica. For this purpose, a survey was conducted among a group of entrepreneurs using the "snowball" sampling method. This method made it possible to identify various aspects of these enterprises, such as, the years of operation and the conditions of formality or semi-formality in which they have developed. Information was also obtained on these business initiatives and their entrepreneurs, such as the gender of the latter, the number of people they employ, the existence or not of government support or whether these businesses are the main source of family subsistence for their managers and, if they have taken out loans, to identify they level of indebtedness. On the other hand, we inquired about the type of clients or the effects they experienced because of COVID- 19 irruption. The results showed that it is young, qualified women between 25 and 33 years old who are the driving force behind these very varied enterprises.
\end{abstract}

Keywords: solidarity economy; female entrepreneurship; local development; endogenous growth.

\title{
Introducción
}

El objetivo de este estudio consiste en analizar una serie de características territoriales y socioeconómicas de emprendimientos situados en los distritos de Sabanillas y San Ignacio de Acosta, en Costa Rica. Estos han sido diseñados principalmente a partir de la teoría del crecimiento endógeno, desarrollada por Friedmann y Douglas (1978), Nelson, R. (1999), Romer (1986; 1990) y Lucas (1988). Los aportes señalados lograron identificar una relación significativa entre el desarrollo de un territorio y la acumulación de factores y capacidades que se crean desde "adentro".

En Veciana (2005), se logró reconocer que los elementos endógenos necesarios en la creación de una empresa son la identificación de una oportunidad de negocio; los factores de producción existentes (recursos materiales, inmateriales y humanos); el entorno de mercado en el que esta se va a desarrollar y, no menos importante, la estrategia de negocio que se utiliza. Además, el marco institucional es el elemento que unifica estas condiciones endógenas para su impulso.

Asimismo, Messina y Hochsztain (2015) señalaron en su estudio las condiciones indispensables para el éxito de los emprendimientos. Entre estas, el acceso a financiamiento y el trabajo independiente se propusieron como los factores que determinan una mayor prosperidad de un negocio o, en otras palabras, como las condiciones para reducir al máximo sus probabilidades de fracaso. Por otra parte, en la investigación de Morales et al. (2015), se descubrió que el factor espacial es significativo, ya que aquellas empresas más cercanas a los centros urbanos tenían mayores posibilidades de desarrollo. Además, se determinó que la motivación y capacitación, especialmente, de los jóvenes emprendedores es crucial para disminuir la "tasa de mortalidad" de los negocios.

La importancia de estudiar el cantón de San Ignacio de Acosta radica en que, aunque es considerado como zona rural, posee una relativa cercanía con el centro urbano de la capital. Este hecho le otorga ventajas en la comercialización de productos y servicios, así como facilita la movilidad de las personas que laboran en esa región.

Ahora bien, hay dos distritos dentro de Acosta que llaman la atención y que demuestran niveles de desarrollo distinto. De acuerdo al último Índice de Desarrollo Social (IDS), 
publicado por el MIDEPLAN (2017), el distrito de San Ignacio registra un IDS catalogado como medio, mientras que Sabanillas, un IDS de 42, que corresponde a un índice muy bajo. De igual forma, en relación con las necesidades insatisfechas, el cantón de San Ignacio posee el mayor nivel de necesidades satisfechas del distrito, mientras que el cantón Sabanillas se ubica en el nivel más alto de necesidades insatisfechas de todo el distrito (ver Figura 1).

\section{Figura 1}

\section{Indicadores de condiciones de vida según cantón/distrito para el cantón de Acosta}

(Censo 2011)
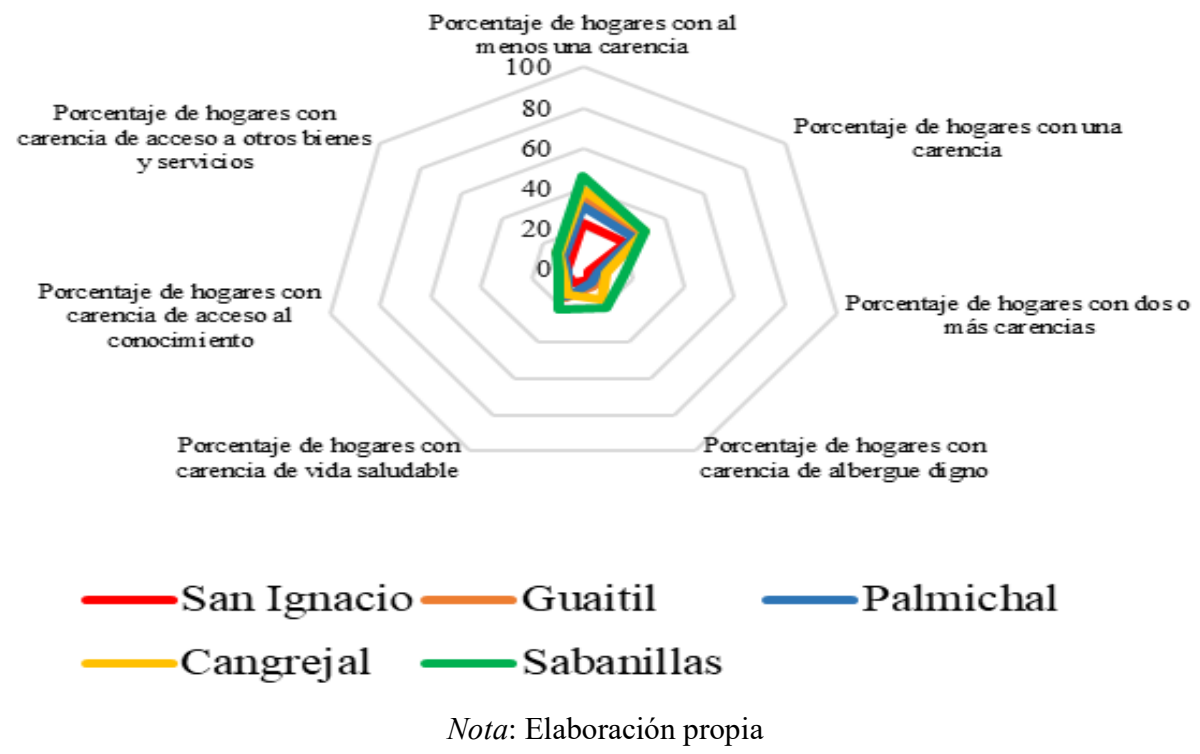

Estos indicadores revelan la necesidad de crear mayores capacidades internas o endógenas, sobre todo, en Sabanillas debido al evidente rezago en su desarrollo con respecto al resto de cantones del mismo distrito. Por el contrario, en términos generales, el cantón de Acosta evidencia mayor potencial para el impulso de la actividad emprendedora por sus características productivas. Según datos de la Municipalidad de Acosta (2007), este cantón presenta una diversificación productiva, principalmente concentrada en el sector primario y terciario.

La creación de emprendimientos en la región y el fortalecimiento de los ya existentes pueden mejorar las condiciones de vida de la población, especialmente, de aquellas personas que cuentan con un nivel menor de escolaridad, pues, tal como lo menciona Castro et al. (2015), las personas de menor cualificación perciben un menor nivel de ingreso, experimentan mayores niveles de insatisfacción de necesidades y se enfrentan a múltiples barreras de acceso al mercado laboral. Hay que recordar que esta situación se agudiza dentro la población femenina.

Por lo tanto, a través de un estudio de caso acerca de los emprendimientos, sobre todo, en zonas rurales, esta investigación se propone contribuir con el fortalecimiento de los negocios de los cantones de San Ignacio y Sabanillas de Acosta mediante la identificación de sus capacidades endógenas.

En primera instancia, se brinda una base teórica sobre el crecimiento endógeno, las características territoriales y socioeconómicas para el desarrollo local. A continuación, se expone información detallada sobre la metodología utilizada. Luego, se presentan los resultados obtenidos y se procede a su discusión. Finalmente, se ofrecen las respectivas conclusiones. 


\section{Materiales y Métodos}

La metodología que se utilizó tiene un enfoque mixto, pues se emplearon variables cualitativas y cuantitativas (Pole, 2009), lo cual facilitó la descripción de las capacidades endógenas de los emprendedores de los distritos de San Ignacio y Sabanillas. Cabe destacar que esta investigación corresponde a un estudio de caso realizado durante el periodo 20192020 .

Para acceder a la información, se acudió a fuentes primarias y secundarias. Por otro lado, además de una amplia revisión documental, se reunieron datos de los emprendedores y se recogió la opinión de los funcionarios de los Gobiernos locales. Por último, se consultaron documentos sobre política e institucionalidad, formas de financiamiento para emprendimientos, trámites, costos y otros elementos relacionados con la formalización de empresas en Costa Rica.

Con el objeto de definir la muestra de los emprendimientos analizados, se utilizó la modalidad de muestreo no probabilístico, en la que la elección de los elementos no depende de la probabilidad, sino de las causas, las características o los propósitos que responden a las propias necesidades de la investigación (Hernández, 2014). Se seleccionó como unidad muestral a los emprendedores de la zona identificados mediante la técnica de muestreo por bola de nieve. Esta técnica sirve cuando los participantes potenciales son difíciles de encontrar. Esto es muy frecuente en el caso de los estudios acerca de emprendimientos, ya que es muy difícil hallar registros dada su naturaleza semiformal o informal. Por este motivo, se encontraron los emprendimientos de la muestra con las características requeridas, gracias a los contactos que nos facilitaron algunos emprendedores y personas de la comunidad. En la selección, se consideraron aquellos emprendimientos que tenían más de 5 años de creación, además de la clasificación que se utiliza en Costa Rica para definir los tipos de negocios según el número de empleados que posee una empresa (Tabla 1).

\section{Tabla 1}

Clasificación de empresas según el número de trabajadores

\begin{tabular}{|l|c|}
\hline \multicolumn{1}{|c|}{ Tipo de empresa } & Empleados \\
\hline Microempresa & Hasta 5 empleados \\
\hline Pequeña empresa & De 6 a 30 empleados \\
\hline
\end{tabular}

Nota: Elaboración propia

Asimismo, se utilizó la clasificación de pyme informal y semiformal, tal como se indica a continuación:

Brenes y Bermúdez (213) definen las pymes informales como:

Las que no formalizan su actividad porque el costo representa un riesgo que no se puede permitir, ya que se caracterizan por la movilidad, la prueba o la operación previa. $\mathrm{Su}$ origen y lógica es la subsistencia, su crecimiento es prácticamente nulo y por ello operan en casas de habitación, lo que promueve su invisibilidad. (p. 103)

Por su parte, para estos mismos autores, las pymes semiformales corresponden a: 
La MYPE semiformal: formaliza lo mínimo necesario para seguir operando, su origen generalmente está relacionado con un proyecto para generar recursos adicionales (no de subsistencia) y su lógica de funcionamiento es de acumulación económica, son más visibles, tienen patente, operan fuera de la casa. (p. 104)

Para los distritos de San Ignacio y Sabanillas de Acosta, se logró definir una muestra total de 37 emprendimientos. A continuación, en la Tabla 2 se detalla la distribución.

\section{Tabla 2}

Tamaños de Fuente para Artículos

\begin{tabular}{|l|c|c|}
\hline \multicolumn{1}{|c|}{ Tipo de negocio } & San Ignacio & Sabanillas \\
\hline Formal/Semi formal & 18 & 4 \\
\hline Informal & 13 & 2 \\
\hline Total & $\mathbf{3 1}$ & $\mathbf{6}$ \\
\hline \multicolumn{2}{|c|}{ Fuente: Elaboración propia }
\end{tabular}

En el desarrollo de esta investigación se diseñaron 2 instrumentos para recoger información. En primera instancia, se realizó una encuesta dirigida a los emprendedores que conformaron la muestra. Esta encuesta consta de 33 ítems con preguntas cerradas y semiabiertas, que se refieren a características básicas como sexo, tipo de residencia, edad, entre otros.

También se consulta sobre características propias de los emprendimientos, como el tipo de emprendimiento, el número de trabajadores, la motivación del negocio, las patentes y el financiamiento. Se incluyeron preguntas relacionadas con la situación ocasionada por el COVID-19 y su afectación a la actividad económica. Esta encuesta se envió de forma electrónica a través del programa Google forms, y se utilizó los programas SPSS y Excel para el procesamiento y visualización de los datos recopilados.

Se elaboró además una entrevista dirigida a los funcionarios de la municipalidad o Gobierno local de Acosta, la cual estuvo conformada de 20 preguntas relacionadas con los distritos, las instituciones u organizaciones públicas y los factores que limitan o potencian el emprendimiento, entre otros. La información de las entrevistas se procesó con ayuda del programa Excel, y su análisis se realizó por medio de gráficos y tablas.

\section{Discusión teórica}

En el siguiente apartado se realizará una revisión de la perspectiva teórica que se planteó para abordar la investigación, que estuvo relacionada con elementos socioeconómicos fundamentales que permitieron caracterizar el territorio. Posteriormente, se analizaron aspectos vinculados con las características propias de los emprendimientos, a partir de la noción de crecimiento endógeno. En el siguiente apartado, se abordará precisamente este modelo de crecimiento endógeno. Finalmente, se describirán las estrategias o los diferentes modelos de emprendimiento que se orientan a la creación de capacidades desde "adentro".

\section{Elementos territoriales: el territorio, la región económica y sus elementos}

El desarrollo local se fundamenta en una vasta red de actores y elementos que coexisten y conviven dentro de un territorio. El territorio constituye la columna vertebral que sostiene los procesos que poseen incidencia sobre el accionar de la sociedad (Esparcia y Noguera, 1999). La noción territorial también incluye el término "región económica", que vincula el territorio 
con los factores productivos, es decir, la tierra, el capital y el trabajo. Desde esta perspectiva, el territorio se convierte en una figura que facilita la producción (Álvarez y Acevedo, 2010).

Las teorías sobre el desarrollo local o territorial integran elementos de enfoque espacial y geográfico. Por ejemplo, el modelo de localización de O'Kelly y Bryan (1996) incluye la localización como una variable clave en la fijación de precios y en la acumulación de factores. Asimismo, el enfoque de la nueva geografía económica de Fujita y Krugman (2004) proporciona una explicación acerca de las formas de aglomeración o clusters presentes en la economía de diversos espacios geográficos.

El desarrollo de un territorio de acuerdo con Hirschman (1958), además de sus condiciones geográficas, requiere encontrar la combinación óptima de los recursos, además, de relacionar estos con las capacidades diseminadas o mal utilizadas. A partir de estas nociones, surge la importancia de los encadenamientos productivos, que se refieren al valor que poseen las decisiones de inversión que este encadenamiento produce en el producto y los "derrames" que genera, es decir, los estímulos adicionales que produce en la economía.

Desde esta perspectiva, se identifican los encadenamientos "hacia atrás" y "hacia delante". Los primeros de estos describen al tipo de inversión que promueve el desarrollo de las materias primas o semiprocesadas utilizadas en la fabricación de un producto final (Pino, 2004, p. 72). Por su parte, los encadenamientos "hacia adelante" se originan cuando "una producción determinada (A) que actúa como input para otra producción (B) opera como estímulo para un tercer producto $(\mathrm{C})$, que puede servir también en calidad de input para el producto (A)" (Pino, 2004, p.72).

\section{Elementos sociales, institucionales y actores relevantes en la creación de capacidades "hacia dentro"}

La participación ciudadana, los agentes de desarrollo e instituciones y la participación de diversos actores son clave para el desarrollo (Miriam y Fernández, 2012). Para alcanzar un nivel óptimo de desarrollo, se debe alentar no únicamente la competitividad empresarial de la unidad productiva o empresa (nivel micro), sino el de todo el entorno. El desarrollo es un proceso sistémico, que incluye diversos agentes, como organizaciones, gobiernos (nivel nacional y local), cooperativas, sistema financiero, organizaciones sin fines de lucro y asociaciones de desarrollo, entre otros.

El ámbito institucional es un elemento transversal que influye en la forma en cómo se organiza el territorio y la interacción social. Esta dimensión tiene que ver con temas como las tradiciones, valores y la identidad construida. Hay que resaltar que la actividad productiva impacta, por ejemplo, en las relaciones comerciales, la confianza, las formas de gobernanza, etc. Las instituciones establecen el marco de incentivos (leyes y normas) en el que se desenvuelve el aparato productivo y social. Coase (1994) afirma que la actividad productiva influye incluso en la "razón de ser" de las empresas.

\section{Elementos y características de los emprendimientos desde un enfoque de desarrollo endógeno}

En el emprendimiento endógeno se origina un proyecto determinado donde todos los actores aportan sus ideas, criterios, capacidades y esfuerzos para su desarrollo (Ospina, 2018).

Como características importantes en el desarrollo productivo y el tejido empresarial, se destaca el "entorno", entendido este como el conjunto de factores sociales, culturales e 
históricos que han configurado el desarrollo de la sociedad. Furlan (2010), Sanabria, Pedraza y Hurtado (2014) destacan los siguientes factores:

1. Los diversos vínculos (formales e informales) entre las empresas, y entre estas y las instituciones.

2. Los trabajadores, porque son la célula del sistema de producción local y son quienes posibilitan el aprendizaje y la innovación.

3. Las relaciones de las empresas de diversos tamaños, pues de estas se derivan diversas relaciones de cooperación. Este punto es en especial relevante, ya que, a partir, de las interacciones empresariales se crean las redes de innovación con las cuales se aprovecha el recurso técnico y la experiencia.

4. Las inversiones exógenas que fortalecen la capacidad local, es decir, el financiamiento para emprendimientos.

5. Programas o estrategias de incubadoras empresariales.

6. Programas o políticas que incentiven el capital humano, la formación y la capacitación.

\section{Modelo de desarrollo o crecimiento endógeno}

De acuerdo con Capello (2011), este modelo plantea el crecimiento en función de la organización del territorio como sistema que vincula elementos socioeconómicos y culturales. Asimismo, hay componentes que son cruciales para el proceso de cambio, como lo son la capacidad emprendedora, los factores de producción locales (trabajo y capital), el capital relacional y el conocimiento (capital humano).

De acuerdo con Rubio (2002), el modelo de crecimiento endógeno contrasta con la teoría neoclásica, pues rechaza el hecho de que los países con un nivel de renta per cápita más bajo crecerán hasta alcanzar el de los países desarrollados. En este este contexto, la teoría del crecimiento endógeno explicará esa falta de convergencia introduciendo variables como el capital humano, input complementario del capital físico en la función de producción. Por su lado, Jiménez (2010) considera para el modelo de crecimiento los siguientes postulados:

1. La tasa de crecimiento del producto per cápita es positiva sin necesidad de suponer que alguna de las variables crece continua y exógenamente.

2. La tasa de crecimiento está determinada por el volumen de acumulación de recursos. En economías con mayor nivel de ahorros, existe mayor crecimiento.

3. La relación capital producto está determinado mediante el parámetro A.

4. No existe el estado estacionario.

5. No hay relación entre la tasa de crecimiento y el nivel de ingreso nacional.

6. A es lo que se conoce como residuo de Solow, y está determinado dentro de la ecuación de crecimiento.

Por lo tanto, para explicar de forma breve el modelo endógeno, se parte de un modelo básico tipo Cobb Douglas, que introduce el capital humano en sus variables:

$$
\mathrm{Y}=\mathrm{AK} \mathrm{K}^{\alpha} \mathrm{H}^{\beta} \mathrm{L}^{1-\alpha-\beta}
$$

Donde Y corresponde al nivel de producto; A representa el progreso técnico; $\mathrm{K}$ es el nivel de capital físico; $\mathrm{L}$, el de trabajo y $\mathrm{H}$, el de capital humano. En términos per cápita, la función de producción sería:

$$
\mathrm{y}=\mathrm{Ak}^{\alpha} \mathrm{h}^{\beta}
$$


Aplicando logaritmos, la función se expresa en tasa de crecimiento:

$$
\mathrm{y}^{*}=\mathrm{a}^{*}+\alpha \mathrm{k}^{*}+\beta \mathrm{h}^{*}
$$

Ahora bien, la acumulación de capital físico y de capital humano es la siguiente:

$$
\begin{aligned}
& \mathrm{k}^{*}=\mathrm{s}_{\mathrm{k}} \mathrm{k}-\left(\mathrm{n}+\delta_{\mathrm{k}}\right) \mathrm{k} \\
& \mathrm{k}^{*}=\mathrm{sh}_{\mathrm{h}} \mathrm{h}-\left(\mathrm{n}+\delta_{\mathrm{h}}\right) \mathrm{h}
\end{aligned}
$$

Donde s es la proporción de producto que se ahorra y $\delta$ es la tasa de depreciación. Sustituyendo esa expresión en (2), se obtiene:

$$
\mathrm{y}^{*}=\mathrm{a}^{*}+\alpha\left[\mathrm{s}_{\mathrm{k}} \mathrm{k}-\left(\mathrm{n}+\delta_{\mathrm{k}}\right) \mathrm{k}\right]+\beta\left[\mathrm{sh}_{\mathrm{h}} \mathrm{-}\left(\mathrm{n}+\delta_{\mathrm{h}}\right) \mathrm{h}\right]
$$

De esta forma, el modelo endógeno indica que la tasa de crecimiento de la renta está en función de la tasa de progreso tecnológico, el crecimiento del nivel de capital físico y humano, de la tasa de depreciación de ambos y del nivel de ahorro. El desarrollo se consigue por medio de la acumulación de capacidades, pues estas elevan el nivel de educación y de conocimiento de las personas, lo que aumenta, a su vez, el valor agregado, logrando así romper con el esquema de los rendimientos marginales decrecientes.

\section{Resultados y Discusión}

\section{Características territoriales de Acosta y los distritos de San Ignacio y Sabanillas}

De acuerdo con los enfoques teóricos analizados, es fundamental considerar las condiciones de caminos y la geografía del territorio para pensar en su desarrollo. Por este motivo, se valoró la calidad de los caminos con base en el material de construcción. Según los datos recogidos en la entrevista realizada al alcalde de Acosta (N. Hidalgo, comunicación personal, 2021), San Ignacio posee en su mayoría caminos de asfalto, que se califican como "altos" en función de su calidad. También se encontraron caminos de lastre, que, por su calidad, se calificaron como "medios". Asimismo, en menor medida, existen caminos de cemento. Por su parte, Sabanillas calificó como baja la calidad de los caminos de asfalto y cemento, pero reportó una calificación media en lastre y tierra.

Por otra parte, se consultó si las organizaciones se encuentran situadas en los distritos de estudio, ya que esto permitió conocer la disponibilidad y el acceso a una gama de servicios, que, al mismo tiempo, potencia la actividad comercial y productiva. Sabanillas resultó ser el distrito que más carece de acceso a servicios básicos, por lo que sus pobladores deben trasladarse (especialmente a San Ignacio) para realizar ciertas gestiones o trámites. San Ignacio es el distrito con mayor desarrollo y hay una amplia gama de servicios disponibles para la población.

Según el alcalde de Acosta, entre los factores que han limitado el desarrollo del cantón están la ausencia de innovación, la falta de cobertura en los servicios (en el caso de Sabanillas), el escaso apoyo gubernamental, el pobre potencial exportador, la poca visibilidad del cantón, la ineficiencia o inexistencia de políticas sectoriales o territoriales y la baja escolaridad.

Sobre los efectos del COVID-19 y su incidencia en el cantón, se indicó que, entre las diversas afectaciones, se destacan la pérdida de ingreso en el sector productivo, el cierre total de algunos negocios, el aumento de despidos, los recortes de la jornada laboral, la disminución 
en la recaudación de impuestos y el aumento de padecimientos relacionados con la salud mental de los ciudadanos.

Sobre el ecosistema emprendedor, Hidalgo señaló que entre las principales limitaciones para emprender en el cantón se pueden mencionar las siguientes:

- Falta de capital inicial

- Poco financiamiento o acceso al crédito productivo

- Ausencia de asesoría en negocios

- Algunas razones relacionadas con la discriminación, por ejemplo, de género y de edad, entre otros.

- Falta de innovación

- Escasez de nuevas ideas

- Poco apoyo gubernamental

- Mala orientación o inexistencia de políticas sectoriales de desarrollo

Con relación a las características productivas, San Ignacio posee una economía basada en el comercio y los servicios, principalmente; es el distrito con mayor densidad urbana de Acosta. Cuenta con una extensión, aproximada, de 23,19 Km2. De acuerdo con el último censo realizado en Costa Rica (2011), existen alrededor de 8961 personas residentes (Municipalidad de Acosta, 2021).

Según, N. Hidalgo, el acceso que San Ignacio posee a los servicios básicos (transporte público, salud, educación, etc.) es bueno. Por otro lado, un $65 \%$ de la actividad productiva se dedica al sector terciario; un 30\%, a la agricultura y la ganadería, y un 5\%, al sector secundario. En el sector primario resalta la producción de cítricos, el cultivo de café y los productos derivados de la apicultura (calificación alta). De importancia media, se calificó la ganadería de engorde y la producción avícola. Finalmente, con calificación baja, se ubicó la ganadería lechera, el cultivo de hortalizas y frijoles.

Sobre la comercialización de los productos mencionados, estos se comercializan, principalmente, dentro del cantón y al resto de la GAM, que comprende las conurbaciones de San José, Alajuela, Cartago y Heredia en Costa Rica). La exportación no es un rubro que se destaque.

Sobre el desarrollo del sector secundario, se señaló que, en general, es muy bajo en el cantón de Acosta. Por el contrario, el sector terciario reportó una alta importancia, sobre todo, en aquellas actividades del sector relacionadas con la salud humana y asistencia social. Como de media satisfacción, se identificaron las actividades de enseñanza, comercio, transporte y almacenamiento, información y comunicaciones, y las actividades relacionadas con labores financieras y de seguros. Por último, las actividades con baja calificación en este sector correspondieron a las de alojamiento y alimentación, actividad inmobiliaria, actividades profesionales y científicas, de apoyo administrativo y artísticas y recreativas.

Ahora bien, al analizar los datos en el caso de Sabanillas, el alcalde Hidalgo comentó que el acceso a servicios básicos es regular. Un 70\% de las actividades que se realizan en este distrito, corresponden al sector primario y el 30\% restante, al de sector servicios. En el sector primario se destaca el cultivo de cítricos, de café y la producción apícola, todos los cuales son clasificados como de alta importancia. De importancia media se clasificó la ganadería de engorde, la producción de huevos y el cultivo de frijoles. Con baja calificación, al cultivo de hortalizas. El sector secundario se clasificó como bajo casi nulo. 
En el sector de servicios se clasificaron como alto las actividades de atención y salud humana; como media, la enseñanza, y como baja, el comercio, el transporte, el almacenamiento, las actividades de alojamiento y alimentación, la información y las comunicaciones, las actividades de seguros e inmobiliarias, entre otros.

\section{Características de los emprendedores de San Ignacio y Sabanillas}

De los emprendimientos encuestados, el 16\% pertenecen a Sabanillas, y el 84\%, a San Ignacio. En total, de los negocios contactados, el $73 \%$ de sus dueños son mujeres y un $27 \%$ son hombres.

Con respecto al rango de edad de los emprendedores, se detectó que oscila entre los 23 y 65 años. No hubo una alta concentración en un rango de edad específico, no obstante, en su mayoría los emprendedores tienen 25, 28, 36, 27, 32 y 33 años, es decir, jóvenes. En relación con sus estudios o nivel académico, los datos determinaron que el $61 \%$ cuenta con un título universitario completo; el $25 \%$, con secundaria finalizada, y el $14 \%$, con secundaria incompleta.

Sobre la cobertura del seguro social, se pudo identificar que en la actualidad un $89 \%$ se encuentra asegurado, en tanto un $11 \%$ reportó no estarlo. Es importante mencionar que de las personas que poseen seguro social, el $85 \%$ reside en San Ignacio y el 15\%, en Sabanillas. Por su parte, de los no asegurados, el $75 \%$ reside en San Ignacio y el 25\%, en Sabanillas.

En lo que concierne a sus núcleos familiares, un 54\% de los emprendedores indicó estar soltero; un $19 \%$, en unión libre y un $27 \%$, casado. Asimismo, un $35 \%$ se identificó como el sostén principal del hogar, mientras que un $54 \%$ aporta ingresos a la familia, mas no como su principal fuente de ingresos; y el $11 \%$ no aporta nada. La mayor parte de los emprendedores $(81 \%)$ reside en casa propia; un 5\%, en residencia y alquilada, y el restante $14 \%$, en casa prestada o en la de sus padres.

En relación con el número de los trabajadores que laboran en los emprendimientos, el $46 \%$ de estos cuenta con un único empleado, que es el mismo dueño; un 30\%, con dos trabajadores. En estas dos situaciones se encuentran la mayor parte de los negocios estudiados. Ahora bien, este dato de acuerdo al distrito, revela que en sabanilla el emprendimiento que cuenta con más trabajadores tiene 4 empleados, mientras que en San Ignacio el que más tiene alcanza 9 empleados. A continuación, en la Tabla 3 se expone el detalle de esta distribución en datos absolutos.

\section{Tabla 3}

Distribución de emprendimientos por distrito

\begin{tabular}{|l|c|c|c|}
\hline Número trabajadores & Sabanillas & San Ignacio & Total general \\
\hline 1 & 1 & 16 & 17 \\
\hline 2 & 4 & 7 & 11 \\
\hline 3 & 0 & 3 & 3 \\
\hline 4 & 1 & 1 & 2 \\
\hline 6 & 0 & 1 & 1 \\
\hline 8 & 0 & 2 & 2 \\
\hline 9 & 0 & 1 & 1 \\
\hline
\end{tabular}

Fuente: Elaboración propia 
Sobre las actividades que realizan los emprendimientos, se constató que estas corresponden a una gran variedad de negocios. A continuación, se detalla el listado de tipos de emprendimiento registrados, según el tipo de actividad que realizan (Tabla 4). Es importante resaltar que entre estas sobresalen aquellas relacionadas con el arte y la confección de artesanías, entre las cuales se pueden incluir las academias de arte, diseño gráfico y artesanos en general.

\section{Tabla 4}

Total de emprendimientos distribuidos por actividad y distrito

\begin{tabular}{|l|c|c|}
\hline Actividad & Sabanillas & San Ignacio \\
\hline Actividades relacionadas con el arte y las artesanías & 1 & 11 \\
\hline Comercio en general, abarrotes & 0 & 1 \\
\hline Cuidado personal o de la salud & 1 & 7 \\
\hline Insumos industriales, agrícolas o ferreteros & 3 & 0 \\
\hline Otros & 0 & 2 \\
\hline Ropa o sastrería & 0 & 2 \\
\hline Servicios de alimentación & 1 & 3 \\
\hline Venta de productos agrícolas, carnes o mariscos & 0 & 5 \\
\hline
\end{tabular}

Fuente: Elaboración propia

Asimismo, se destacan los emprendimientos relacionados con la salud y cuidado personal: salones de belleza, manicuristas, elaboración de jabones, venta de maquillaje y medicinas. También se identificaron emprendimientos orientados a brindar servicios de alimentación, como sodas y restaurantes, entre otros. En relación con las patentes de los emprendimientos, El 58\% (12 negocios) cuentan con algún tipo de estas patentes. El 25\% cuenta con permisos que les otorgan derecho comercial, venta de alimentos o licor, y un $17 \%$ no detalla el tipo de patente que posee. Solo 4 de estos negocios se ubican en Sabanillas y 8, en San Ignacio.

Con respecto a la comercialización que realizan estos emprendimientos, un $17 \%$ que comercializa al menudeo está ubicado en Sabanilla y un $83 \%$ de estos, en San Ignacio. En tanto que el comercio realizado a través de intermediarios, un $89 \%$ está localizado en San Ignacio, y un 11\%, en Sabanillas. Un 29\% del comercio que se realiza de forma directa por intermedio de empresas u otras organizaciones se sitúa en Sabanillas, y un 71\%, en San Ignacio. Esta distribución tiene mucho sentido, ya que el distrito de Sabanillas se especializa en la comercialización de insumos ferreteros y agrícolas, que constituyen materia prima para otros negocios.

Sobre la motivación de los negocios, 16 de los emprendimientos señalaron que empezaron su negocio motivados al identificar una oportunidad de negocio; otros 9 de estos lo hicieron por necesidad o desempleo; 5 , como complemento de su fuente de ingresos primaria, y 7 , movidos por otras razones, como la independencia económica y tradición, entre otros.

También se indagó sobre los proveedores de los emprendimientos. La mayor parte de estos acuden como su principal fuente de provisión a tiendas, pulperías o bazares locales, seguido de distribuidoras o fábricas, mientras que la minoría se abastece de la oferta de otros emprendedores de la zona: supermercados o ellos mismos elaboran su materia prima (Figura 2). 


\section{Figura 2}

Cronograma de Ensayo de Prueba de Campo

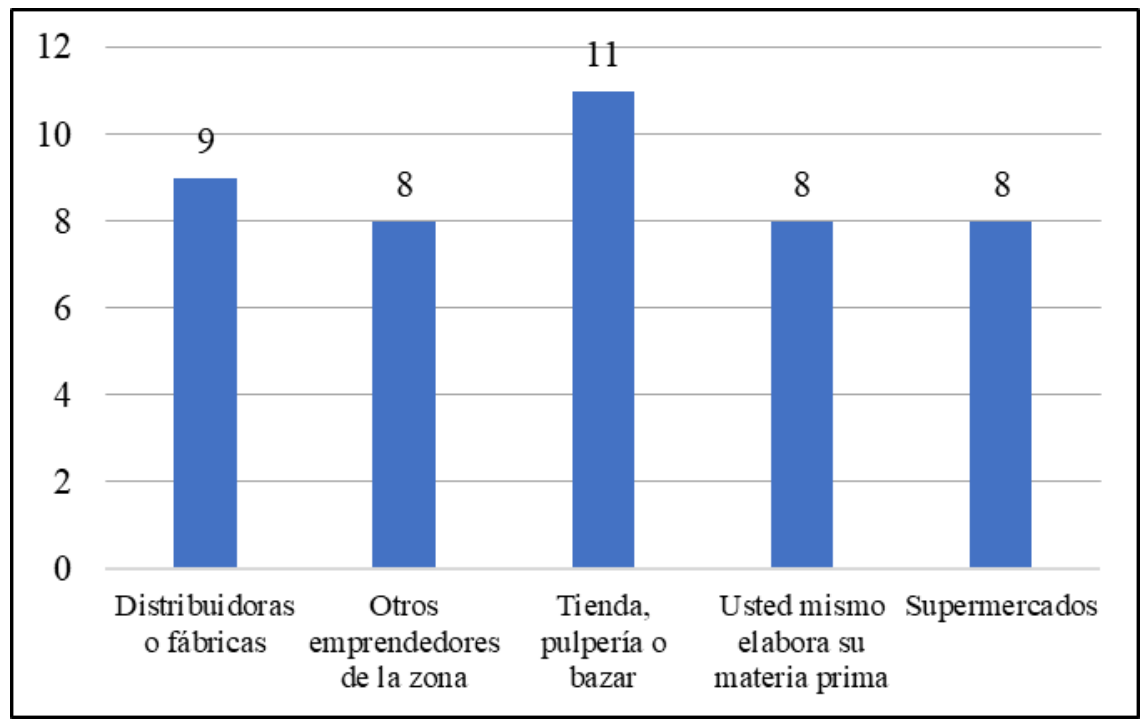

Nota: un emprendimiento puede comprar a varios proveedores de manera simultánea. Fuente: Elaboración propia.

En términos generales, el comportamiento productivo de estos emprendimientos evidencia que un encadenamiento productivo hacia atrás, ya que se demandan bienes finales para la producción. Asimismo, como ya se mencionó antes, la mayor parte de los negocios $(46 \%)$ se componen de un único trabajador, con lo cual la generación de empleo se basa en un esquema mayoritariamente de autoempleo.

Por otra parte, atendiendo a la situación exógena ocasionada por el COVID-19 en el país y su impacto significativo en muchas variables macroeconómicas, se consideró necesario consultaren la encuesta a los emprendedores sobre el impacto que ha tenido este factor en el volumen de venta de sus negocios. Un 45\% de estos reportó que sus ventas habían disminuido entre un $15 \%$ y un $25 \%$. En tanto que un $20 \%$ señaló pérdidas en sus ventas entre un $25 \%$ y $50 \%$. Finalmente, un $20 \%$ indicó que sus ventas registraron un descenso de más del $50 \%$ (Figura 3).

\section{Figura 3}

Distribución porcentual de emprendimientos de acuerdo con la disminución en ventas

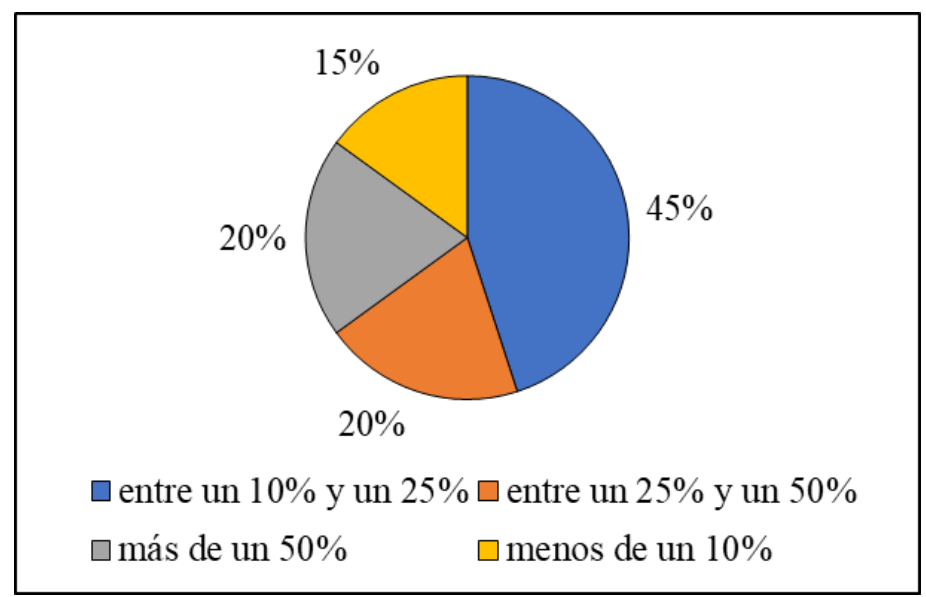

Fuente: Elaboración propia. 
Con respecto al número de despidos de trabajadores o suspensión de jornadas que generó el contexto pandémico, no se evidenció un alto índice. Solo 3 emprendimientos ubicados en San Ignacio reportaron haber despedido o suspendido a trabajadores. Hay que recordar que la mayoría de los emprendimientos son unipersonales.

Asimismo, se consultó si recibieron algún tipo de apoyo o de flexibilidad crediticia, para afrontar las pérdidas económicas ocasionadas por la pandemia. Solo dos emprendimientos de la zona de San Ignacio reportaron apoyo de la banca.

Todos los emprendedores conocen la tramitología para la formalización.

\section{Discusión de resultados}

En el caso de San Ignacio y, en términos generales, el cantón de Acosta identificó como factor favorable para la comercialización y el intercambio de bienes de bienes y servicios su ubicación geográfica cercana a otras zonas de la GAM. Esta ventaja espacial coincide con lo planteado por O'Kelly y Bryan (1996), quienes identifican la localización como un elemento importante de desarrollo, incluso, San Ignacio de Acosta ha demostrado una configuración productiva acorde con su ubicación dentro del país, en la medida que ha logrado cambiar su composición ocupacional, orientada esta hacia las actividades del sector primario.

Un factor externo identificado como favorable corresponde al esfuerzo que se ha realizado en Costa Rica, en el año 2014, por normar y establecer procesos que orienten a los emprendedores. Entre estos aportes se pueden identificar el establecimiento de una política de fomento al emprendimiento y el manual del emprendedor (MEIC, 2014; 2019), lo que ha representado un acicate para el desarrollo endógeno vinculado con la capacidad institucional del país y como respuesta a la necesidad de crear, mantener o fortalecer los emprendimientos. Esto corrobora el enfoque planteado por Miriam y Fernández (2012), en donde se señala que el factor institucional y político es el encargado de amalgamar estas capacidades endógenas y empujar la reactivación económica de un territorio.

Ahora bien, el estudio permite identificar como factor de éxito el alto nivel académico de algunos emprendedores (la mayoría con título universitario). Esto representa un elemento importante en la teoría del desarrollo endógeno por la importancia que tiene el capital humano en el desarrollo de las actividades productivas, como bien lo ha planteado Becker (2009). Además, a este factor hay que sumarle el conocimiento derivado de la experiencia en el emprendimiento que potencia aún más estas capacidades.

Actualmente, San Ignacio es el distrito con mayor actividad económica de Acosta. De igual manera, el acceso a los servicios básicos es calificado de bueno. También se ha valorado en términos positivos la calidad de sus caminos y calles. Este elemento es fundamental para el crecimiento económico según la tesis propuesta por Álvarez y Acevedo (2010), que plantea que la dotación de factores y la existencia de estas organizaciones impulsa la actividad económica, la educación y la salud, entre otros elementos necesarios en la mejora de la calidad de vida de sus pobladores.

Como amenazas internas, se destacan el escaso incentivo hacia la innovación, el bajo apoyo gubernamental, las pocas fuentes de financiamiento y las dificultad de acceder a créditos productivos. Esto coincide con la tesis que propone Fonseca y Chaves (2014), que afirma que los negocios en Costa Rica no nacen con la expectativa de innovar, pues no existen los incentivos suficientes para ello. 
También se identifican como desventajas el poco potencial exportador y la escaza presencia de encadenamientos productivos "hacia adentro". Esto, en contraste con el modelo de desarrollo endógeno, es una señal de alerta, ya que parte de la capacidad de crear, diversificar y sobrevivir de los negocios se basa en sus oportunidades de innovar y en la capacidad que tengan de contar con un capital de inversión inicial, así como posibilidades de internacionalizarse por medio de las exportaciones.

En el caso del distrito de Sabanillas se identificó las mismas oportunidades y amenazas de factores externos señalados para San Ignacio. Sobre los factores internos como oportunidades, se destaca la presencia de emprendedores con niveles educativos altos (la mayoría con título universitario), el desarrollo especialmente de actividades enmarcadas en el sector primario: ganadería, cultivo de cítricos, frijoles y café, entre otros.

Además, cabe mencionar algunas amenazas o limitantes para el desarrollo del emprendimiento productivo, sobre todo, "hacia adentro" en Sabanilla: la dificultad para monitorear emprendimientos dentro de estas zona, el poco incentivo y asesoría a la innovación; la ausencia casi por completo de apoyo gubernamental y de fuentes de financiamiento, la carencia de un potencial exportador en el cantón, las dificultades de acceso a los servicios básicos calificado como regulares y el estado de las vías de transporte, clasificadas como regulares a malas.

Sobre las oportunidades en este distrito, se identificó que son muy similares a las definidas para San Ignacio, pues existe un bajo nivel de endeudamiento de los emprendedores, en su mayoría mujeres; el adecuado conocimiento sobre la formalización y el patentamiento de los negocios, además, de las motivaciones para crear un negocio, entre las que se destacan el deseo de alcanzar independencia económica y generar ingresos como principal fuente de subsistencia.

\section{Conclusiones}

Costa Rica ha experimentado una desaceleración económica, especialmente desde 2018 en adelante, hasta alcanzar una caída de su Producto Interno Bruto (PIB) en $-4,5 \%$ para el año 2021 (BCCR, 2020). A esto hay que sumarle la tasa de desempleo que alcanzó el $20 \%$ en el año mencionado; un aumento del déficit primario y la situación coyuntural ocasionada por el COVID-19 (INEC, 2020), que ha afectado la economía a nivel internacional.

Específicamente sobre el cantón de Acosta se puede concluir que es un cantón cercano a la GAM, lo que le otorga ciertas ventajas, especialmente en la comercialización de productos. Por su parte, San Ignacio se destaca por ser el distrito con mayor crecimiento económico y mejor desempeño comercial, entre otras características. Este distrito cuenta con caminos y calles calificadas en buen estado, un acceso adecuado a los servicios básicos, la presencia de muchas organizaciones e instancias que potencian el proceso de desarrollo, como centro de salud, bancos, cooperativas, centros comerciales, seguridad y asociaciones de desarrollo, entre otros.

En el ámbito productivo, este cantón está orientado principalmente hacia las actividades de servicios y comercio, no obstante, la actividad primaria no deja de desarrollarse, en especial la de cultivo de cítricos. La actividad industrial es casi nula.

En el caso de Sabanillas, destacan su menor crecimiento económico, la presencia de caminos calificados como regulares, del mismo modo que el acceso a los servicios básicos. La actividad productiva principal de este distrito es la actividad agropecuaria. 
En relación con los emprendimientos, sus dueños son jóvenes, con alto nivel académico (título universitario alcanzado), con motivaciones claras de emprender negocios (una idea $\mathrm{u}$ oportunidad), con gran una gran capacidad de diversificar sus negocios, aunque, sobre todo, orientados a la venta de productos y a la oferta de servicios de cuidado personal. En su mayoría, las dueñas de este tipo de negocios son mujeres emprendedoras. Además, se evidencia una escasa capacidad para diversificar las exportaciones, así como un poco apoyo de financiamiento y afectación en las ventas por el COVID-19.

En Costa Rica, existen diversas herramientas promovidas por la última política definida para el fomento del emprendimiento, sin embargo, no existe una hoja de ruta o acciones estratégicas que brinden una guía para realizar acciones encaminadas a desarrollar los emprendimientos, especialmente, aquellos que requieren un soporte tecnológico o innovadora. Tampoco hay un verdadero marco de incentivos ya sea por intermedio de crédito o asesoría.

Finalmente, se observa que los trámites de formalización del emprendimiento en Costa Rica, aunque no representan necesariamente un costo alto, son un desincentivo para que las personas formalicen sus emprendimientos, pues este proceso demanda mucho tiempo por su complejidad.

\section{Referencias}

Álvarez, R., y Acevedo, J. (2010). El territorio como factor del desarrollo. Semestre Económico, 13(27), 39-62.

Banco Central de Costa Rica (BCCR). (2020). Programa Macroeconómico 2019-2020.

Becker, G. S. (2009). Human capital: A theoretical and empirical analysis, with special reference to education. University of Chicago press.

Brenes, L., y Bermúdez, L. (2013). Semiformalidad de la mediana y pequeña empresa (MIPYME) en Costa Rica y su relación con la competitividad y el desarrollo. UNED Research Journal, 4(1), 101-116.

Caja Costarricense del Seguro Social (CC. SS.). (24 de mayo de 2020). CC.SS. Clasificación MiPymes. https://www.ccss.sa.cr

Capello, R. (2011). Location, regional growth and local development theories. Aestimum, 58, 1-25.

Castro, J. M., Garita, J., \& Odio, M. (2015). Análisis sobre la Dinámica de Transición y Duración del Desempleo en Costa Rica. Revista de Ciencias Económicas, 32(2), 39.

Coase, R. (1994). La empresa, el Mercado y la Ley. Madrid. Alianza.

Esparcia, J., y Noguera, J. (2000). Reflexiones en torno al territorio y al desarrollo rural. El desarrollo rural en la Agenda, 9-44.

Fonseca, R., y Chaves, M. C. (2014). Política Pública, "Costa Rica Emprende": Un análisis de los resultados. Revista de Relaciones Internacionales, 93-110.

Friedmann, J. y Douglas, M. J. (1978). Agropolitan development: toward a new strategy for regional planning in Asia, en F. Lo y K. Salih (eds.), Growth Pole Strategy and Regional Planning Development Policy. Oxford: Pergamon

Fujita, M., y Krugman, P. (2004). La nueva geografía económica: pasado, presente y futuro. Investigaciones Regionales, (4), 177-206.

Furlan, A. (2010). Actuar en la crisis: el sistema eléctrico en la perspectiva del desarrollo endógeno. Análisis de caso de la costa atlántica bonaerense, Argentina. Nadir: revista electrónica de geografía austral, 2(2). 
Hernández, R. (2014). Metodología de la investigación. México: McGRAW-HILL.

Hidalgo, F. (2021). [Comunicación personal] 12 de mayo 2021.

Hirschman, A.O. (1958). The Strategy of Economic Development. USA, Yale.

Instituto Nacional de Estadística y Censo (INEC). (diciembre, 2020). Encuesta Nacional de Hogares [Base de datos]. Recuperado de https://www.inec.cr/encuestas/encuesta-continua-de-empleo

Instituto Nacional de Estadística y Censo (INEC). (2011). Censo Nacional de Costa Rica.

Jiménez, F. (2010). Crecimiento económico: Enfoques y modelos. Capítulo 5 - Teoría del crecimiento endógeno. IDEAS Working Paper Series from RePEc, IDEAS Working Paper Series from RePEc, 2010.

Lucas, R. E. (1988): On the mechanics of economic development. Journal of Monetary Economic, 22, 1:129144

Messina, M., y Hochsztain, E. (2015). Factores de éxito de un emprendimiento: Un estudio exploratorio con base en técnicas de data Mining. Tec empresarial, 9(1), 30-40.

Ministerio de Economía Industria y Comercio (MEIC). (2014). Política de fomento al emprendimiento 20142018

Ministerio de Economía Industria y Comercio (MEIC). (2019). Manual para la Persona Emprendedora en Costa Rica.

Ministerio de Planificación Económica (MIDEPLAN). (2017). Índice de Desarrollo Social 2017.

Miriam, G., y Fernández, Q. (2012). Los actores en el desarrollo económico local. Un estudio en América Latina. Perspectivas, 15(30), 71-116.

Morales-Jiménez, J., Bustamante-González, Á., Vargas-López, S., Pérez-Ramírez, N., y Sereno-Pareja, O. (2015). Factores de éxito emprendedor en dos municipios de la montaña de Guerrero, México. Nova scientia, 7(15), 416-435.

Municipalidad de Acosta. (2007). Información General sobre el Cantón de Acosta.

Nelson, R. (1999): How New is New Growth Theory? Challenge, 40, 5:29-58.

O'Kelly, M., y Bryan, D. (1996). Agricultural location theory: von Thunen's contribution to economic geography. Progress in Human geography, 20(4), 457-475.

Ospina, D. (2018). Construcción de comunidades colaborativas desde el diseño y el emprendimiento endógeno. Revista Escuela de Administración de Negocios, (84), 63-77.

Pino, O. (2004). Análisis de encadenamientos productivos para la economía regional, base 1996. Theoria, 13(1).

Pole, K. (2009). Diseño de metodologías mixtas. Una revisión de las estrategias para combinar metodologías cuantitativas y cualitativas Renglones (60) http://hdl.handle.net/11117/252

Romer, M. P. (1986): «Increasing returns and long run growth». Journal of Political Economy, 94:1002- 1037

Romer, P. M. (1990). Endogenous technological change. Journal of political Economy, 98(5, Part 2), 71-102.

Rubio, C. H. (2002). La teoría del crecimiento endógeno y el comercio internacional. Cuadernos de estudios empresariales, (12), 95.

Sanabria, S., Pedraza, P., y Hurtado, E. (2014). El emprendimiento como fuente de desarrollo y fortalecimiento de las capacidades endógenas para el aprovechamiento de las energías renovables. Revista Escuela de Administración de Negocios, (77), 152-167. 
Veciana J.M. (2005). La creación de empresas Un enfoque gerencial, Barcelona. p.312. https://www.uv.es/creaemp/Veciana2005Libro.pdf 